\title{
S11-02
}

\section{FAMILIAL RISK FACTORS FOR THE COURSE OF BIPOLAR DISORDER}

M. Preisig ${ }^{1}$, F. Ferrero ${ }^{2}$

${ }^{1}$ Department of Psychiatry, University Hospital Center and University of Lausanne, Prilly, ${ }^{2}$ Department of Psychiatry, University Hospital, Geneva, Switzerland

Aims: The major aims of the present paper were to:

1. assess associations between the course of bipolar-I disorder in probands and the presence and course characteristics of mood disorders in their relatives and

2. assess associations between manic and depressive symptoms in probands and relatives.

Methods: A family study including 125 bipolar-I patients and all available first-degree relatives has been conducted at two Swiss sites. All participants were evaluated using the Diagnostic Interview for Genetic Studies. Assessed course variables included the age of onset, the number of episodes and social functioning in terms of GAF scores. Among the 237 interviewed relatives of bipolar probands, 32 also exhibited bipolar disorder.

Results: The occurrence of bipolar disorders in relatives was not associated with course variables in bipolar probands, whereas the occurrence of unipolar depression in relatives was associated with a more favorable course in probands in terms of higher lifetime GAF scores. Regarding the expression of symptoms during episodes, associations between disorders in probands and relatives were observed for dysphoric and psychotic rather than for typical manic symptom patterns.

Conclusion: Our data did not provide support for a significant association between the course of bipolar disorder and the presence or the course characteristics of bipolar disorders in relatives. Surprisingly, the presence of unipolar depression in relatives was even associated with a better course of the bipolar disorder in probands. Nevertheless, bipolar disorder revealed some degree of similarity across family members, particularly regarding the expression of dysphoric and psychotic symptom patterns. 